\title{
Manipulation of Small Parts Being Assembled on a Horizontally Vibrating Plate
}

\author{
Kristina LIUTKAUSKIENË*, Sigitas KILIKEVIČIUS**, Ramūnas ČESNAVIČIUS***, \\ Bronius BAK ̌̌YS $* * * *$, Linas PAUKS̆TAITIS*****
}

\author{
*Kaunas University of Technology, Studentu 56, 51424 Kaunas, Lithuania, E-mail: kristina.liutkauskiene@ktu.lt \\ **Kaunas University of Technology, Studentu 56, 51424 Kaunas, Lithuania, E-mail: sigitas.kilikevicius@ktu.lt \\ ***Kaunas University of Technology, Studentu 56, 51424 Kaunas, Lithuania, E-mail: ramunas.cesnavicius@ktu.lt \\ ****Kaunas University of Technology, Studentu 56, 51424 Kaunas, Lithuania, E-mail: bronius.baksys@ktu.lt \\ *****Kaunas University of Technology, Studentu 56, 51424 Kaunas, Lithuania, E-mail: linas.paukstaitis@ktu.lt \\ crossref http://dx.doi.org/10.5755/j01.mech.25.5.22827
}

\section{Introduction}

During recent years in manufacturing and engineering the importance of automatic assembly was significantly increasing. To implement this task, robots, manipulators and other assembly machines are usually used equipped with various active, passive or hybrid assembly control systems [1-3].

One of the most effective methods is vibratory assembly which can be divided into two main stages: alignment and mating of parts. During the alignment stage, the dynamic directionality effect occurs due to the asymmetry of vibratory excitation, and the parts can be easily manipulated in order to achieve a required alignment and positioning precision.

Currently horizontally vibrating planes are mostly applied for transportation of parts. Frei et al. [4, 5] presented a line to transport multiple objects along different trajectories. The plane of the line consists of separate segments vibrating both in the horizontal and vertical directions allowing to align, sort out and transport the parts at different velocities along different trajectories. Bohringer et. al. [6] proposed a mechanism for parts orientation which systematically performs orientation and localization of the parts by exiting elastic vibrations of the plane. Tarasevičius and Fedaravičius [7] analysed the movement of a body on a plane oscillated in a circular motion, when the effective coefficient of dry friction was periodically controlled during each turn of the vibration platform. However, transient regimes of body motion and the dependence of body motion trajectories on the system parameters have not been analysed. Vose et. al. [8] presented a design of a 6-degree-of-freedom mechanisms consisting of a rigid plate coupled to linear actuators through flexures for vibratory manipulation. However, the design and control of such mechanism is quite complicated. Higashimori et al. [9] proposed a method of nonprehensile manipulation via plate vibration, where the two degrees of freedom of a part on the plate are controlled by only one actuator by manipulating the frequency and offset angle of the sinusoidal displacement input to the actuator.

Recently vibrational techniques are investigated intending to fully exploit the advantages of vibratory excitation for assembly and transportation of microparts in microelectromechanical systems (MEMS). Xiao et. al. [10] investigated a large stroke manipulator for micro/nano ma- nipulation on a plane. By using two electromagnetic actuators for each axis, the micromanipulator operates in planar spiral and quadrant motions. Two-dimensional noncontact transportation of small objects in air using flexural vibration of a plate with four ultrasonic transducers and a reflector was analysed by Kashima et. al. [11]. The authors demonstrated that by controlling the phase difference of the two pairs of the transducers, the flexural vibration of the plate and the acoustic standing wave in air could be shifted, which enabled manipulation of small particles in a two-dimensional plane.

This paper proposes a new method of manipulating small parts on a horizontal plane, when the plane is excited in two perpendicular directions by a harmonic law with different frequencies and a phase shift. To determine motion patterns which are most suitable for automatic assembly, a mathematical model of movement of parts on the horizontal plane was developed and the most suitable trajectories for connecting surfaces matching as well as the most suitable excitation parameters for automatic assembling process were obtained.

\section{Equations of part motions}

A positioning error appears during automatic assembly of parts. To compensate this error, a suitable search trajectory pattern can be selected and applied for the parts. In this way, the connective surfaces of the parts will overlap and the parts will be joined (Fig. 1). Search trajectories can take various forms, but the best trajectories are the closed forms, as it is always preferred to move the parts along a closed trajectory.

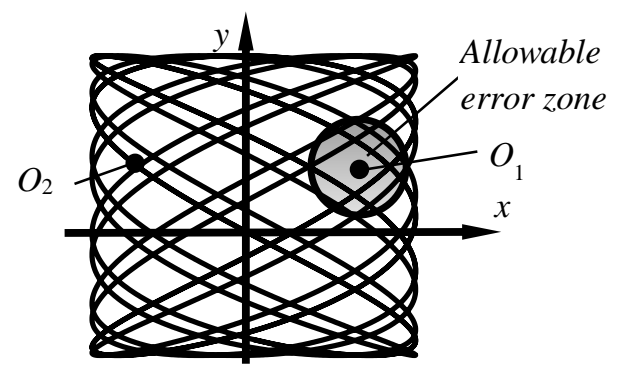

Fig. 1 Search scheme: $O_{1}$ centre of stationary part, $O_{2}$ centre of movable part 
By varying the phase shift and frequency ratio between the vibrations in the $\mathrm{x}$ and $\mathrm{y}$ directions, a series of Lissajous patterns can be obtained. The horizontal plane could be made to move in different motion patterns by controlling the phase shift, frequencies, and amplitudes of the two perpendicular vibroactuators attached to this plane.

Considering this, the motion of a small cylindrical part on a vibrating plate excited in two perpendicular directions is investigated. The height of the part in comparison with the diameter of the part is relatively low, therefore, an assumption is made, that the mass of the part is concentrated in the mass centre. Then, the part may be considered as a flat part. The immovable coordinate system $\xi O \eta$ and movable $x O_{1} y$ coordinate systems are located on the horizontal plane (Fig. 2). The plate is excited in two perpendicular directions by harmonic oscillations of different frequencies. The motion of the plate can be defined as follows:

$$
\left\{\begin{array}{l}
\xi=\xi_{0}+R_{e} \cos \omega_{x} t \\
\eta=\eta_{0}+R_{e} \sin \omega_{y} t
\end{array},\right.
$$

where: $R_{e}$ is the amplitude, $t$ is time, $\omega_{x}$ is the frequency of harmonic oscillations along the $\xi$ axis, $\omega_{y}$ is the frequency of harmonic oscillations along the $\eta$ axis.

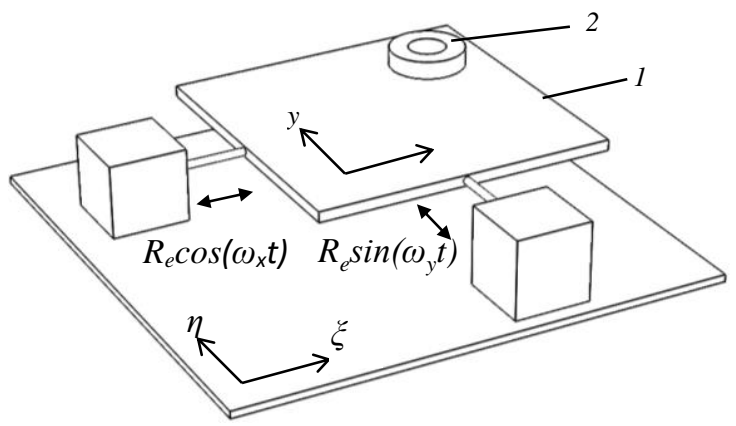

Fig. 2 Schematic view of the part and the plate with coordinate frames: 1 - plate, 2 - cylindrical part

Assuming that the transient plate motion disappears in a short time, to simplify the part motion analysis, only the steady-state plane motion is considered. The differential equations of small part motion on the plate are as follows:

$$
\left\{\begin{array}{l}
\dot{x}+\mu g \frac{\dot{x}}{\sqrt{\dot{x}^{2}+\dot{y}^{2}}}=R_{e} \omega_{x}^{2} \cos \omega_{x} t \\
\dot{y}+\mu g \frac{\dot{y}}{\sqrt{\dot{x}^{2}+\dot{y}^{2}}}=R_{e} \omega_{y}^{2} \sin \omega_{y} t
\end{array},\right.
$$

where: $\mu$ is the coefficient of sliding friction between the part and the plane, $\dot{x}$ and $\dot{y}$ are relative velocity projections of the part in $x$ and $y$ axes directions.

\section{Part positioning}

To solve the differential equations of the part motion, MATLAB software was used. The mathematical modelling showed that the part motion from the initial point to the positioning point is complex, and examples of part's centre motion pattern along the $x$ and $y$ directions are shown in Fig. 3., The trajectories of the motion of the part varies depending on the ratio of excitation frequencies $k=\omega_{x} / \omega_{y}$ (Fig. 4). When the tolerance zone of the assembly is small, not all the trajectories of the motion of the part are suitable for automated assembly (for example Fig. 4, b and d), as these patterns do not fill the entire search area. In this case, the most suitable trajectories for the matching of the surfaces to be assembled are these with the ratio of excitation frequencies $k=2.1 \ldots 2.9$, when the search motion occurred in a closed square, rectangular or circular area. Such complex trajectories of the search motion, when the centre of the part makes the loops in a different location, i.e. the desired area is passed very densely, can be used to match surfaces of parts with very low tolerance zones, i.e. the clearance between the parts to be assembled is small. For accurate and quick matching of connective surfaces of the parts, it is necessary to determine the parameters making an influence on the area of the search motion, because this area predetermines the search zone of the connective surfaces. The modelling demonstrated that as the amplitude of plate oscillations increases, the area of the search motion also increases. When the frequency of plate oscillations increases, the search pattern tends to become denser (the resolution of the search pattern increases).

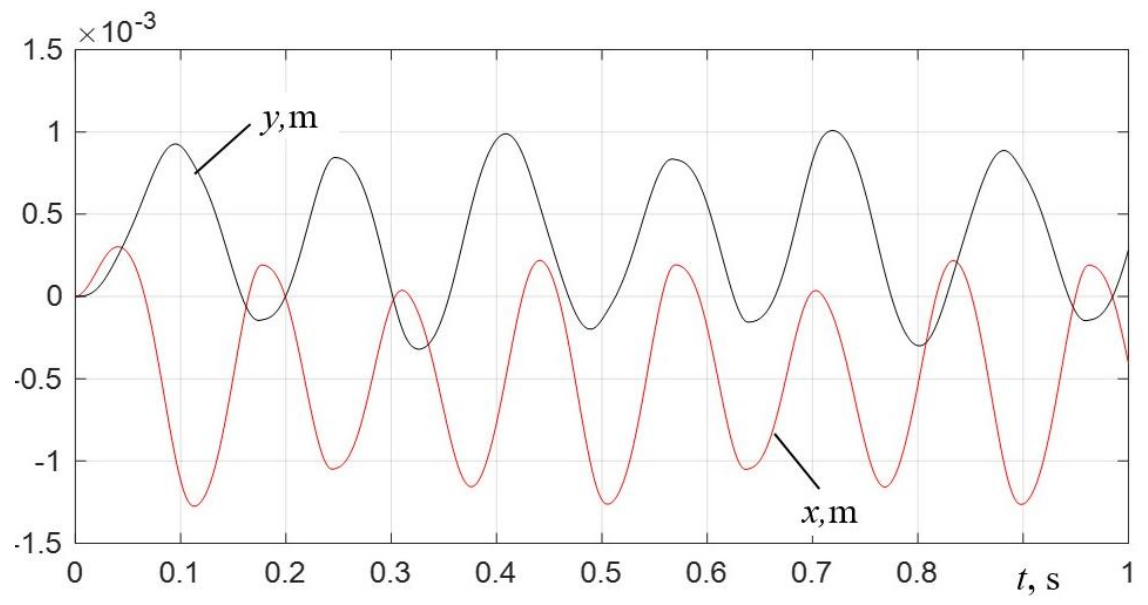

Fig. 3 Characteristic vibration part of the search motion, along $x$ and $y$ directions, as $\mu=0.15 ; \omega_{y}=40 \mathrm{~s}^{-1} ; \omega_{x}=1.2 \omega_{y}$; $R_{e}=0.001 \mathrm{~m}$ 

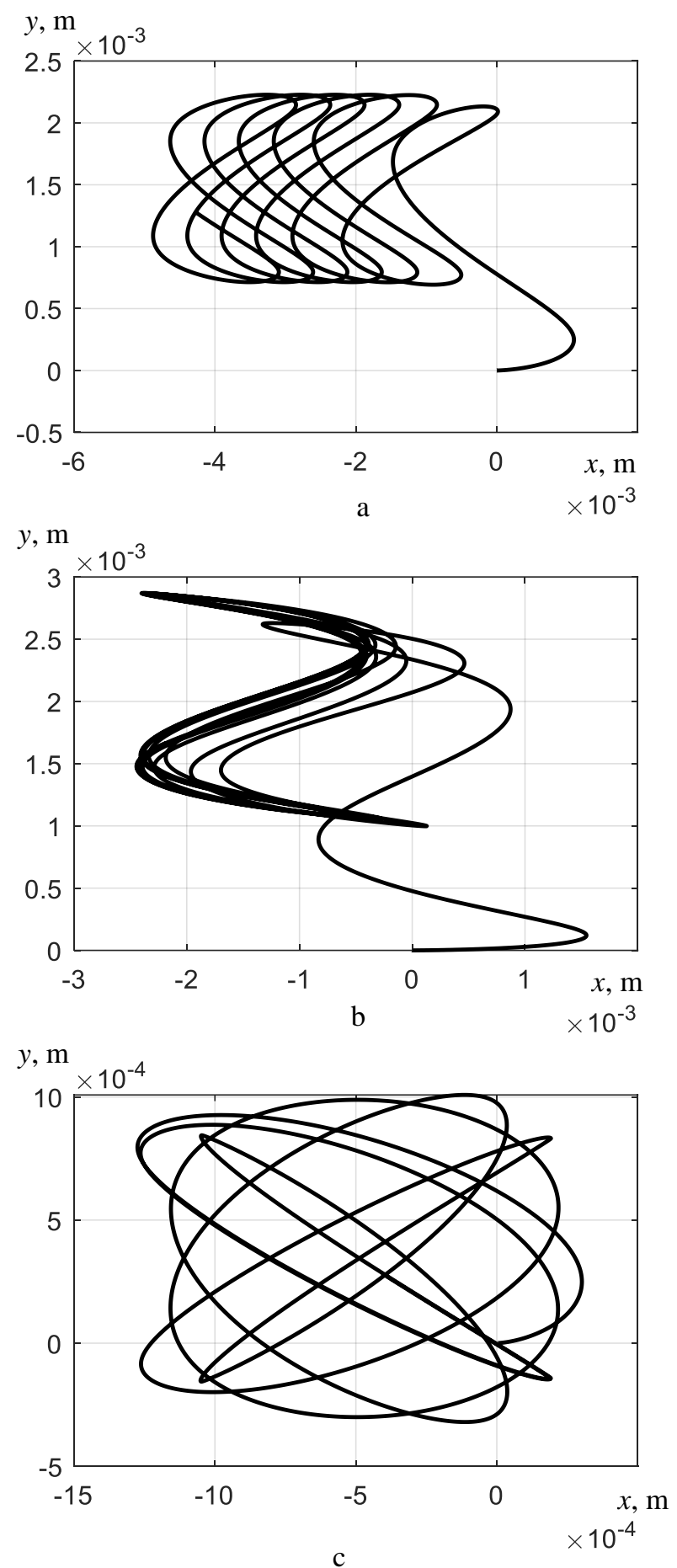
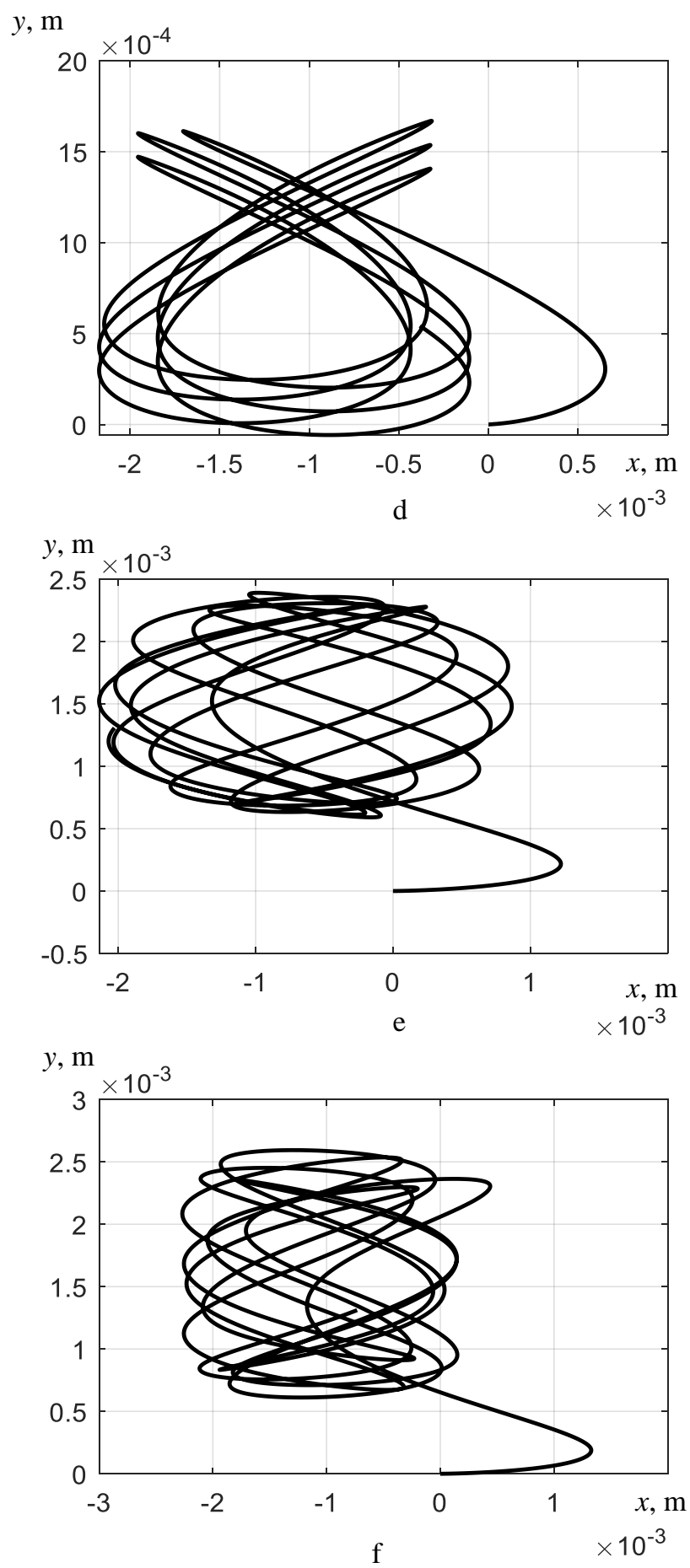

Fig. 4 Complex trajectories of the search motion, as $\mu=0.15 ; \omega_{y}=40 \mathrm{~s}^{-1} ; \omega_{x}=k \omega_{y} ; R_{e}=0.001 \mathrm{~m}: \mathrm{a}-k=2 ; \mathrm{b}-k=3 ; \mathrm{c}-k=1.2$; $\mathrm{d}-k=1.5 ; \mathrm{e}-k=2.2 ; \mathrm{f}-k=2.4$

To obtain a better searching effect, the searching patterns should be distributed homogeneously. The modelling showed that when the frequency difference is equal to $1,\left(\left|\omega_{y}-\omega_{x}\right|\right)=1$, the search trajectory of the part is located in a square shape, and the size of this square is limited by the amplitude of the plate oscillations (Fig. 5). i. e. the square area is $2 R_{e}$ and it does not depend on the frequency or the friction coefficient. The resolution of the search pattern in the area increases as the frequency increases.

To move the part over a greater distance, it is necessary to increase the frequency of the excitation or to reduce the coefficient of friction. The friction coefficient can be reduced by controlling dry friction [12]. It is possible to control the direction of the motion of the part to be assembled by changing the initial phase of excitation signals, [13].

When the plate is provided with excitation of different frequencies along the mutually perpendicular directions, the conditions of parts matching get better. The part moves repeatedly, making loops in different positions, so the part axis would get into the zone of the allowable error. Therefore, the parts to be assembled connect unhindered. 


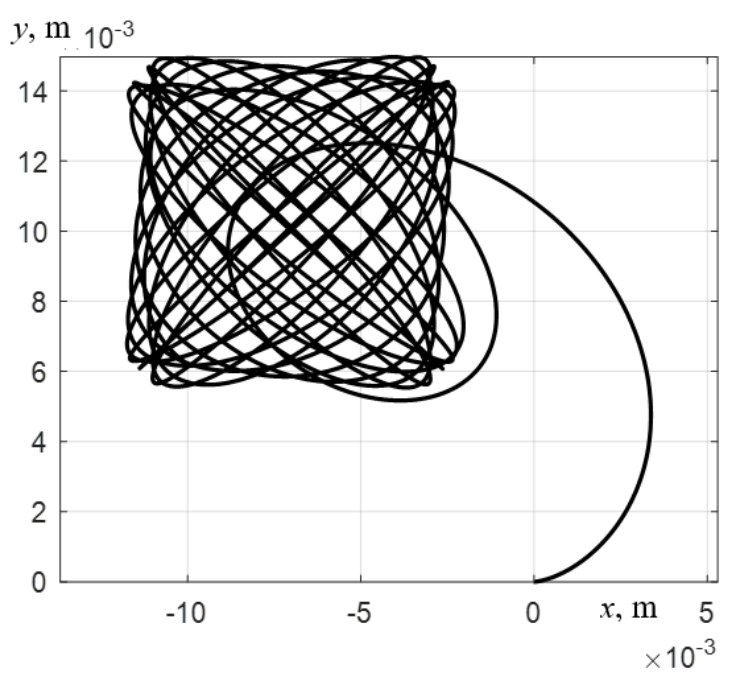

Fig. 5 Complex trajectories of the search motion, as $\mu=0.1$; $\omega_{x}=20 \mathrm{~s}^{-1} ; \omega_{y}=21 \mathrm{~s}^{-1} ; R_{e}=0.005 \mathrm{~m}$

The differential equations of the motion of a small part when the plate is excited in two perpendicular directions with different frequencies are as follows:

$$
\left\{\begin{array}{l}
\dot{x}+\mu g \frac{\dot{x}}{\sqrt{\dot{x}^{2}+\dot{y}^{2}}}=A_{e} \omega_{x}^{2} \cos \omega_{x} t \\
\dot{y}+\mu g \frac{\dot{y}}{\sqrt{\dot{x}^{2}+\dot{y}^{2}}}=B_{e} \omega_{y}^{2} \sin \omega_{y} t
\end{array},\right.
$$

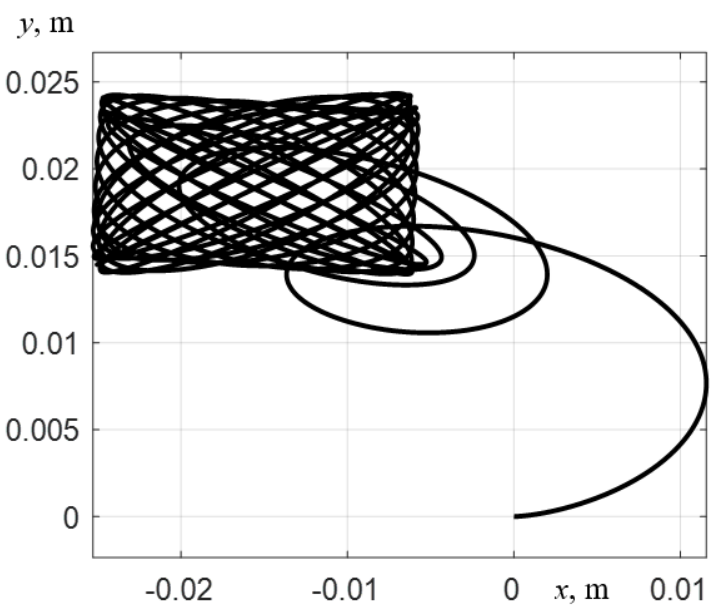

a where $A_{e}$ is the amplitude of harmonic excitation along the $x$ axis, $B_{e}$ is the amplitude of the harmonic excitation along the $y$ axis. Then, the search area takes a rectangular shape (Fig. 6). When the excitation amplitude in the $y$ direction is lower than in the $x$ direction, the motion pattern takes a shape of a rectangle with a width to height ratio higher than 1 , and vice versa, when the excitation amplitude in $y$ direction is greater than in the $x$ direction, pattern arranged in takes a shape of a rectangle with a width to height ratio lower than 1

When the tolerance zone is small, the most effective way to coincide connecting surfaces is to induce the complex search motion trajectories of a square or rectangular shape.

Comparing all these search trajectories, attention should be paid that in automated assembly process the assembly time is very important. It depends on how quickly the part moves during the search as well as the size and shape of the search area. The mathematical modelling demonstrated that when the frequency difference is equal to 1 , it takes more time for the part to move along the entire search area in compare to the cases when the excitation frequency ratio is $k=2.1 \ldots 2.9$.

The plane can be excited in two perpendicular directions with different frequencies with a phase shift.

By combining the frequency ratio and the phase shift, the plane motion developed different Lissajous patterns (Fig. 7).

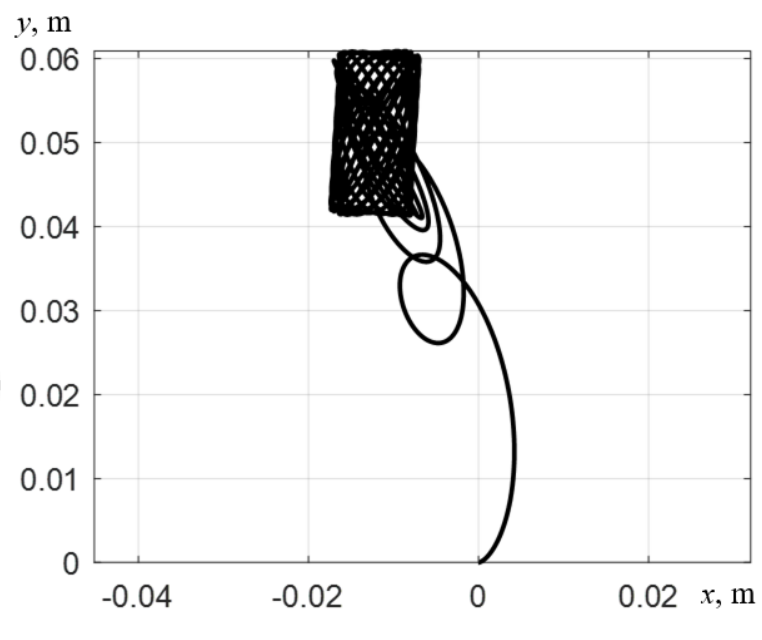

b

Fig. 6 Complex trajectories of the search motion, as $\mu=0.1 ; \omega_{x}=20 \mathrm{~s}^{-1} ; \omega_{y}=21 \mathrm{~s}^{-1}$, where a $-A_{e}=0.01 \mathrm{~m} ; B_{e}=0.005 \mathrm{~m}$; $\mathrm{b}-A_{e}=0.005 \mathrm{~m} ; B_{e}=0.01 \mathrm{~m}$

In this case the motion equations are as follows:

$$
\left\{\begin{array}{l}
\dot{x}+\mu g \frac{\dot{x}}{\sqrt{\dot{x}^{2}+\dot{y}^{2}}}=R_{e} \omega_{x}^{2}\left(\sin \omega_{x} t-\alpha\right) \\
\dot{y}+\mu g \frac{\dot{y}}{\sqrt{\dot{x}^{2}+\dot{y}^{2}}}=R_{e} \omega_{y}^{2}\left(\sin \omega_{y} t-\alpha\right)
\end{array}\right.
$$

In practice, the most common case is where the frequency of excitation in one direction is two times higher than in the other. Then, the trajectory of the movement of the part depends very much on the angle of the phase $\alpha$
(Fig. 8). When $\alpha=\pi / 4$ and $\alpha=3 \pi / 4$, the part moves in trajectories similar to parabolic sections. When $\alpha=0, \alpha=\pi / 4, \alpha=\pi$ and $\alpha=3 \pi / 2$, part stationary motion trajectories are similar to the shape of the number 8 . When the excitation frequency in the $y$ direction is greater than in the $x$ direction, the motion trajectories of the plane become similar to the shape of the infinity sign. This demonstrates that by changing the excitation frequencies, the search trajectories can be controlled.

Using this method for searching, the search area is also equal to $2 R_{e}$ and it does not depend on the frequency or the friction coefficient. The resolution of the search pattern in the area increases as the frequency increases. 

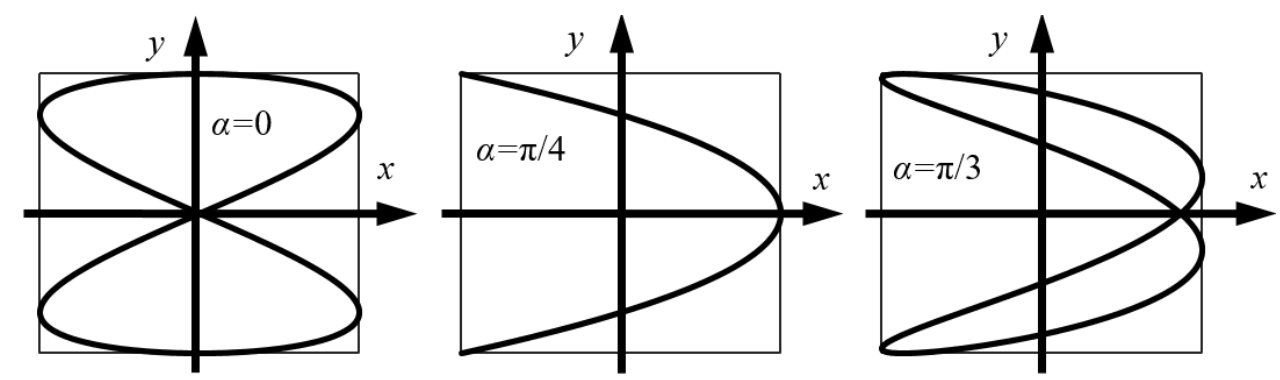

Fig. 7 Plate motion trajectories, depending on the phase shift
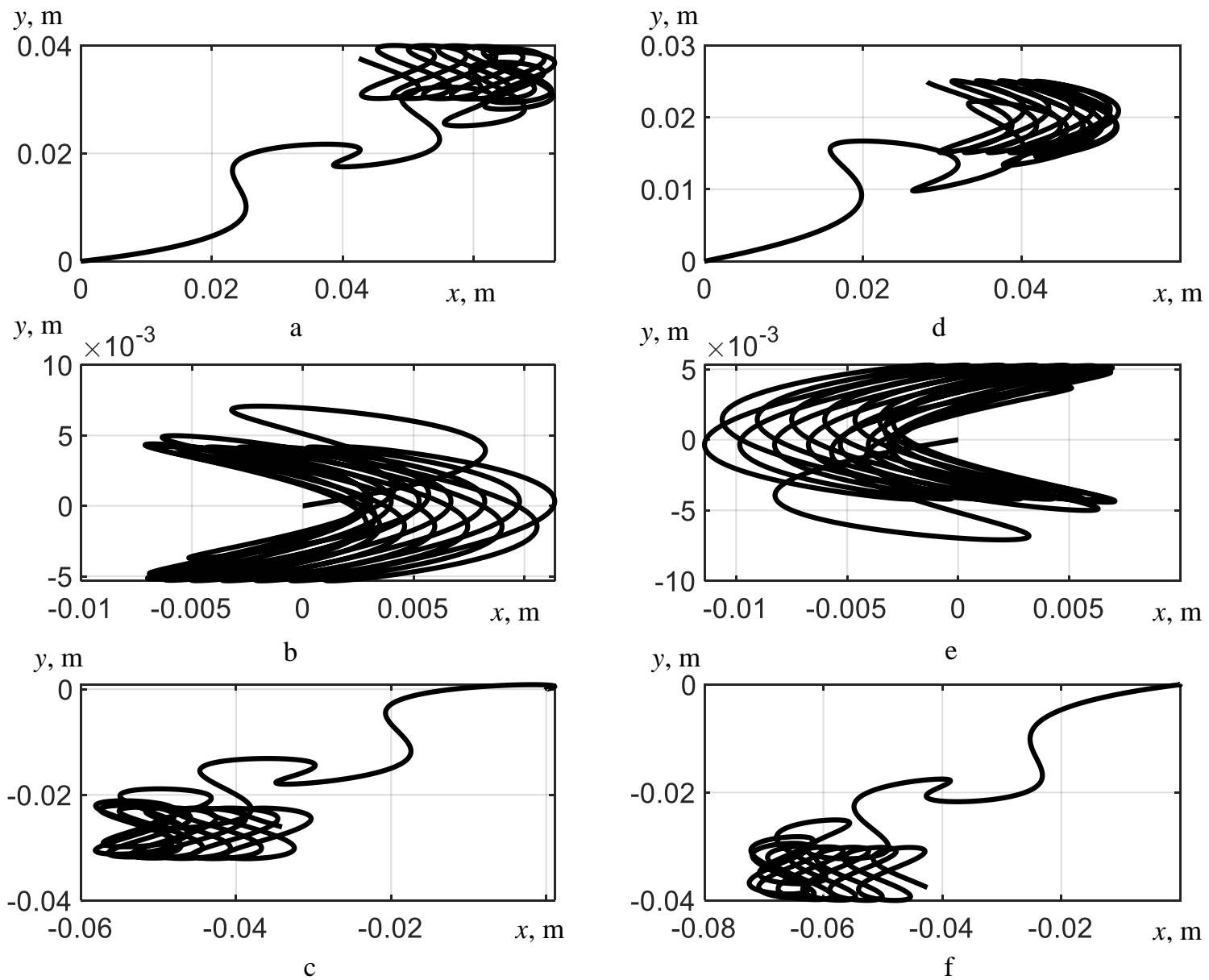

Fig. 8 Trajectories of the search motion, as $\mu=0.15 ; \omega_{y}=20 \mathrm{~s}^{-1} ; \omega_{x}=2 \omega_{y} ; R_{e}=0.01 \mathrm{~m}: \mathrm{a}-\alpha=0 ; \mathrm{b}-\alpha=\pi / 4 \mathrm{c}-\alpha=\pi / 2$; $\mathrm{d}-\alpha=3 \pi / 2 ; \mathrm{e}-\alpha=3 \pi / 4 ; \mathrm{f}-\alpha=\pi$

\section{Conclusions}

A mathematical model of movement of parts on a horizontal plane, when the plane is excited in two perpendicular directions by a harmonic law with different frequencies and a phase shift was developed. The modelling showed that the steady-state motion trajectories of the part are close to the plane motion, which moves along complex curves (Lissajous). By changing the excitation parameters and the coefficient of friction between the part and the plane, the part can be easily moved along prescribed trajectory ensuring a necessary positioning precision and alignment for the assembly operation.

When the speed of surface alignment is relevant, the most suitable trajectories for the matching of assembled surfaces are these with the excitation frequency ratio $k=2.1 \ldots 2.9$.
When the tolerance zone is small, the most effective way to coincide connecting surfaces is to induce the complex search motion trajectories of a square or rectangular shape. i.e. when the frequency difference is equal to one $\left|\omega_{y}-\omega_{x}\right|=1$, as it improves the conditions of accurate matching.

The size of the search area can be controlled by changing the excitation amplitude and the resolution of the search trajectory motion can be controlled by changing the excitation frequency. The transitional search period can be increased or decreased by controlling the coefficient of friction.

This method can be easily used for matching the surfaces of small parts with a small assembly clearance. 


\section{References}

1. Zhang, K.; Shi, M.; Xu, J.; Liu, F.; Chen, K. 2017. Force control for a rigid dual peg-in-hole assembly, Assembly Automation 37(2): 200-207. https://doi.org/10.1108/AA-09-2016-120.

2. Takahashi, J.; Fukukawa, T.; Fukuda, T. 2016. Passive alignment principle for robotic assembly between a ring and a shaft with extremely narrow clearance, IEEE/ASME Transactions on Mechatronics 21(1): 196204. http://dx.doi.org/10.1109/TMECH.2015.2448639.

3. Park, H.; Bae, J. H.; Park, J. H.; Baeg, M. H.; Park, J. 2013, October. Intuitive peg-in-hole assembly strategy with a compliant manipulator, IEEE 44th International Symposium on Robotics (ISR): 1-5. http://dx.doi.org/10.1109/ISR.2013.6695699.

4. Frei, P. U.; Weisendanger, M.; Buchi, R.; Ruf, L. 2000. Simultaneous planar transport of multiple objects on individual trajectories using friction forces, Kluwer Academic Publishers: 49-64. http://dx.doi.org/10.1007/978-1-4615-4545-3_3.

5. Frei, P. U. 2002. An intelligent vibratory conveyor for the individual objects transportation in two dimensions, Proc. Of the 2002 IEEE/RSJ Int. Conf. on Intelligent Robots and Systems EPFL, Lausanne, Switzerland: 1832-1837. http://dx.doi.org/10.1109/IRDS.2002.1044022.

6. Bohringer, K. F.; Bhatt, V.; Goldberg, K. Y. 1995, May. Sensorless manipulation using transverse vibrations of a plate, In Robotics and Automation, Proceedings, 1995 IEEE International Conference on 2: 19891996.

7. Tarasevicius, K.; Fedaravicius, A. 1999. Mechanisms of vibrational transportation with controlled dry friction, Proceedings of tenth world congress on the theory of machines and mechanisms, Oulu, Finland: 21982203.

8. Vose, T. H.; Turpin, M. H.; Dames, P. M.; Umbanhowar, P.; Lynch, K. M. 2013. Modelling, design and control of 6-DoF flexure-based parallel mechanisms for vibratory manipulation, Mechanism and Machine Theory 64: 111-130.

https://doi.org/10.1016/j.mechmachtheory.2012.12.007.

9. Higashimori, M.; Yamaguchi, K.; Shibata, A. 2018. Omnidirectional Nonprehensile Manipulation Using Only One Actuator, Robotics 7(3): 34. https://doi.org/10.3390/robotics7030034.

10. Xiao, X.; Li, Y.; Xiao, S. 2017. Development of a novel large stroke 2-DOF micromanipulator for mi- cro/nano manipulation, Microsystem Technologies 23(7): 2993-3003.

http://dx.doi.org/10.1007/s00542-016-2991-3.

11. Kashima, R.; Koyama, D.; Matsukawa, M. 2015. Two-dimensional noncontact transportation of small objects in air using flexural vibration of a plate, IEEE transactions on ultrasonics, ferroelectrics, and frequency control 62(12): 2161-2168. http://dx.doi.org/10.1109/TUFFC.2015.006998.

12. Baksys, B.; Liutkauskiene, K. 2010. Manipulation of a part on a vibrating plane under controlled dry friction, Mechanika 3(83): 28-34.

13. Baksys, B.; Ramanauskyte, K.; Povilionis, A. B. 2009. Vibratory manipulation of elastically unconstrained part on a horizontal plane, Mechanika 1(75): 36-42.

K. Liutkauskienė, S. Kilikevičius, R. Česnavičius, B. Bakšys, L. Paukštaitis

\section{MANIPULATION OF SMALL PARTS BEING ASSEMBLED ON A HORIZONTALLY VIBRATING PLATE}

S u m m a r y

The article proposes a new method of manipulating parts on a horizontal plane, when the plane is excited in two perpendicular directions by a harmonic law with different frequencies and a phase shift. To determine motion patterns which are most suitable for automatic assembly, a mathematical model of movement of parts on the horizontal plane was developed. The results demonstrated that by changing the excitation parameters and the coefficient of friction between the part and the plane, the part can be easily moved along prescribed trajectory ensuring a necessary positioning precision and alignment for the assembly operation. The most suitable trajectories for connecting surfaces matching as well as the most suitable excitation parameters for automatic assembling process were obtained. The proposed method can be efficiently applied in automatic assembly to match the surfaces of small parts with a small assembly clearance.

Keywords: automatic assembly, vibrations, manipulation, oscillations, positioning.

Received February 28, 2019

Accepted October 14, 2019 